\section{Piezoelectric deformable mirror with adaptive multiplexing control}

\author{
A. N. Simonov, S. Hong, and G. Vdovin \\ Delft University of Technology, \\ Electronic Instrumentation Laboratory, \\ Mekelweg 4, 2628 CD Delft, The Netherlands
}

\begin{abstract}
We describe a simple and efficient implementation of adaptive multiplexing control for high-order piezoelectric deformable mirrors. The relatively high capacitance of piezoelectric actuators allows the electrical charge to be stored in a disconnected actuator, retaining its displacement while the other actuators can be addressed. Adaptive multiplexing, consisting of selective addressing of only those actuators that need to change their elongation in the current cycle, improves the mirror performance and simplifies the driver electronics. In experiment, a 12-channel prototype of a deformable mirror with multiplexing control has been characterized. At appropriate update rates with a fixed set of control signals, the shape of the deformable mirror remains nearly constant. A surface displacement error does not exceed $\sim \lambda / 100 \mathrm{rms}$ at a multiplexing frequency of $700 \mathrm{~Hz}$ with a full interactuator stroke of $\sim 2 \mu \mathrm{m}$. @ 2006 Society of Photo-Optical Instrumentation Engineers.

[DOI: 10.1117/1.2219733]
\end{abstract}

\section{Subject terms: deformable mirror; multiplexing.}

Paper 060053LR received Jan. 20, 2006; revised manuscript received Apr. 13, 2006; accepted for publication Apr. 20, 2006; published online Jul. 5, 2006.

Deformable mirrors $(\mathrm{DM})$ are the key components of adaptive optics (AO) systems. ${ }^{1,2}$ A number of scientific, ${ }^{3,4}$ medical, ${ }^{5,6}$ and industrial ${ }^{2,5,7}$ applications require inexpensive, reliable, and high-quality multichannel DMs. ${ }^{8}$ The number of channels in a DM can reach $10^{2}-10^{3}$, especially in astronomical AO systems. ${ }^{1,2,4}$ Typically, each DM actuator is driven by an individual control unit, resulting in complex electronics and cabling for a high-order DM. This makes the AO system rather bulky, vulnerable to handling, and very expensive. The total cost of the driver electronics for a high-order AO system may account for two-thirds of the total system cost. $^{2}$

An attempt to simplify the DM electronics by sequential addressing of actuators was made by Kibblewhite et al. ${ }^{10}$ They built a 59-channel piezoelectric faceplate mirror that was driven by several high-voltage amplifiers (HVA) by way of 16 high-voltage switches assembled from discrete components. Due to the implementation and control complexity, this approach has not won general acceptance. ${ }^{2}$

In this letter, we report on the possibility of a high-order piezoelectric DM with internal multiplexing and simple driver electronics that implements adaptive addressing. This approach implies that the addressing of piezoelectric actuators is accomplished not continuously, but as needed through a pregenerated lookup table to follow the required DM figure. Only those actuators that must adjust the DM shape are addressed in each cycle. Other actuators are up-

0091-3286/2006/\$22.00 @ 2006 SPIE dated at the slowest possible rate, to keep their size. The simplicity and compactness of the multiplexing electronics allows it to be potentially integrated with the mirror, reducing the complexity of cable interconnections.

In our experiment, we used a 12-channel experimental piezoelectric DM with a 25.4-mm aperture from OKO Technologies. ${ }^{11}$ The mirror uses standard 3.2-mm-diameter and 30-mm-long tubular PZT actuators (produced by PI Ceramic), positioned in a rectangular grid with 7-mm pitch. With a control voltage ranging from 0 to $300 \mathrm{~V}$, the DM full stroke reaches $\sim 7 \mu \mathrm{m}$ (hysteresis $<10 \%$ ), and its interactuator stroke, i.e., the maximum displacement between the adjacent actuators, amounts to $\sim 2 \mu \mathrm{m}$. The actuators have comparatively low capacitance of $C_{a}=12 \mathrm{nF}$, with $1 / e$ leakage time exceeding $30 \mathrm{~s}$ while mounted in the mirror.

The driver electronics for the DM (see Fig. 1) includes a midpower unipolar MOSFET-based HVA, loaded by a 12channel multiplexer. The HVA is capable of driving a single actuator with $500-\mathrm{V}$ amplitude at $18 \mathrm{kHz}$. Actuators were multiplexed using 12 miniature high-voltage optotriacs ( $\left.\mathrm{Q}_{1}-\mathrm{Q}_{12}\right)$ with optically isolated low-voltage controls. A computer-integrated 8-bit D/A converter board was used to generate all the control signals for the mirror operation. Figure 2 shows the simplified timing diagram for 1,2, and 12 channels of the DM. As seen, in a multiplexing period, the voltages $V_{1}$ to $V_{12}$ produced by the HVA are sequentially applied to the DM channels via the optotriac switches $\mathrm{Q}_{1}-\mathrm{Q}_{12}$ activated by channel selection signals.

The operating bandwidth and surface stability are the critical parameters of the multiplexed DM. To characterize the multiplexed DM in open-loop mode, we used the setup shown in Fig. 1. A light beam from a He-Ne laser at $\lambda$ $\cong 633 \mathrm{~nm}$ is collimated and split by a $50 / 50$ beam splitter $\mathrm{BS}_{1}$. Part of the collimated beam illuminates a low-finesse asymmetric Fabry-Pérot interferometer formed by the DM mirror and a second 50/50 beam splitter $\mathrm{BS}_{2}$. The light reflected from the interferometer is picked up by $\mathrm{BS}_{1}$ and goes through the imaging optics (IO) to a CCD for fringe registration, or to a photodiode for measurements of the surface stability.

Limited bandwidth of the driver electronics and mechanical DM resonances reduce the overall DM performance at high frequencies. In each situation, there is a threshold frequency $\left(F_{t h}\right)$ at which the multiplexing leads to interchannel crosstalk and temporal instability of the mirror profile. Figure 3 presents the interferograms of the DM influence function at (a) $F<F_{t h}$ and (b) $F>F_{t h}$. In this experiment, a voltage of $150 \mathrm{~V}$ was applied to the DM channel 8 (see Fig. 1), while all other channels were kept at $0 \mathrm{~V}$. The threshold frequency of multiplexing was found to be $F_{t h} \sim 1.5 \mathrm{kHz}$ without adaptive optimization of actuator addressing.

The DM operation with a large interactuator stroke can be considered as the extreme case of multiplexing. Actually, in this case a high voltage difference $\left(U_{\Delta}\right)$ is required between adjacent DM actuators, and the HVA needs to perform full-amplitude switching at its maximum frequency. This frequency is given (see Fig. 2) by: $N^{-1}\left[t_{H V A}+\left(t_{o n}\right.\right.$ $\left.\left.+t_{o f f}\right) / 2\right]^{-1}$, where $t_{H V A}$ is the HVA settling time, and $t_{o n}$, $t_{\text {off }}$ are the switching times of optotriacs. At $U_{\Delta}=300 \mathrm{~V}$, we have $t_{H V A} \cong 60 \mu \mathrm{s}, t_{o n} \cong 8 \mu \mathrm{s}, t_{o f f} \cong 30 \mu \mathrm{s}$, and $F_{t h}$ reaches 


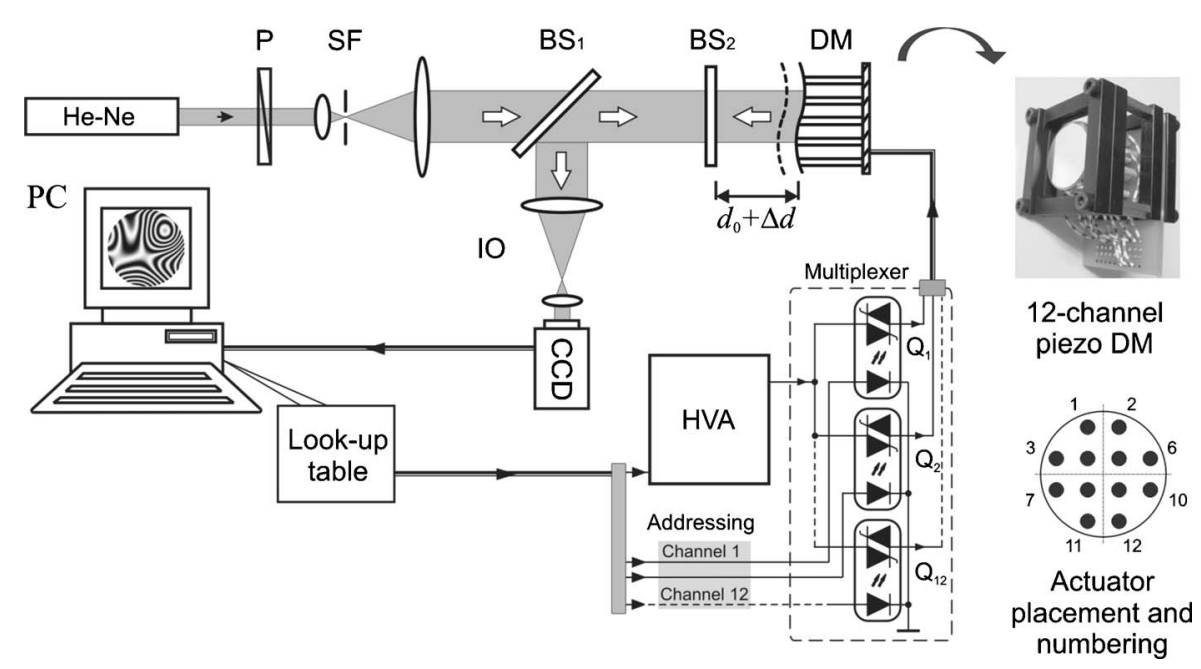

Fig. 1 Experimental setup. P, polarizer; SF, spatial filter; $\mathrm{BS}_{1}, \mathrm{BS}_{2}$, beam splitters; IO, imaging optics; $\mathrm{DM}$, deformable mirror; HVA, high-voltage amplifier; $\mathrm{Q}_{1}-\mathrm{Q}_{12}$, optotriacs.

$\sim 1 \mathrm{kHz}$. The experimental dependence of $F_{t h}$ on $U_{\Delta}$ (and the corresponding interactuator stroke) is shown in Fig. 4. In the experiment, $U_{\Delta}$ was applied to the even channels of the mirror, whereas the odd channels were maintained at $0 \mathrm{~V}$. As seen, a maximum interactuator stroke of $\sim 2 \mu \mathrm{m}$ that corresponds to $U_{\Delta}=300 \mathrm{~V}$ was obtained at $F<F_{t h}=700 \mathrm{~Hz}$. This value is in agreement with the $F_{t h}$ estimate above. A stroke of such magnitude, however, is rarely needed in common $\mathrm{AO}$ applications. At the opposite extreme, the multiplexed DM provides a $\sim 0.1-\mu \mathrm{m}$ interactuator stroke $\left(U_{\Delta}=12 \mathrm{~V}\right)$ at $F_{t h}=3.6 \mathrm{kHz}$. The equivalent Nyquist frequencies (i.e., single-channel bandwidths of the multiplexed DM) are $350 \mathrm{~Hz}$ and $1.8 \mathrm{kHz}$, respectively. These values meet or exceed the requirements for typical atmospheric correction. ${ }^{2,10}$

Figure 4 shows that the maximum update rate $F_{t h}$ can be adjusted, depending on the required DM deformation. For small displacements, the addressing can be carried out at higher frequencies, and vice versa. This allows optimization of the overall mirror performance by choosing an appropriate update rate for each DM channel.

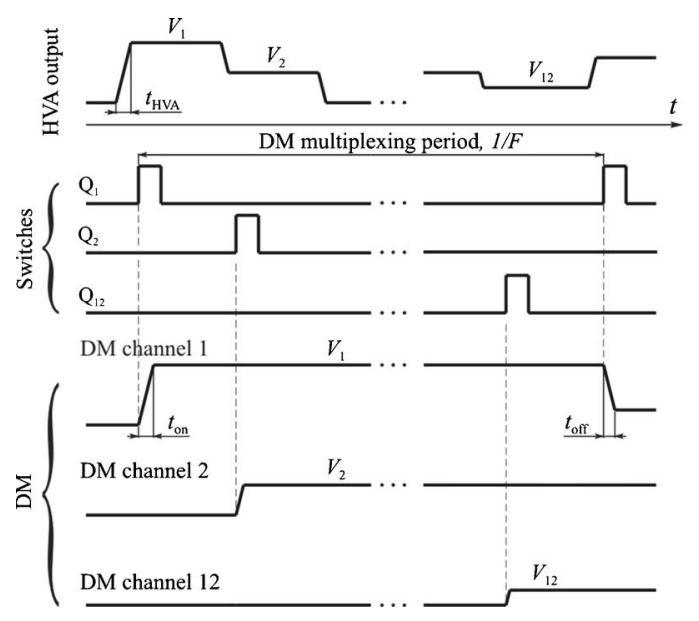

Fig. 2 Simplified timing diagram for 1, 2, and 12 channels of the DM.
To estimate the figure stability of the multiplexed DM, the surface displacement error $(\Delta d)$ was evaluated as a function of $F$ for the DM with a single activated channel. We estimated the measured intensity variation $(\Delta I)$ of the lowest-order fringe of the DM interference pattern (see Fig. 3 ), to determine very small displacements $\Delta d$ of the mirror surface, caused by the interchannel crosstalk. The reflected intensity $(I+\Delta I)$ of an asymmetric Fabry-Pérot interferometer, ${ }^{12}$ can be written as:

$I+\Delta I=I_{0} R\left(d_{0}+\Delta d\right)$

where $R(d)=\left|\frac{-\sqrt{R_{1}}+\sqrt{R_{2}} \exp (2 i k d)}{1-\sqrt{R_{1} R_{2}} \exp (2 i k d)}\right|^{2}$.

$I_{0}$ is the intensity of incident light; $d_{0}$ is the sum of the DM stroke caused by the addressed actuator and the distance between $\mathrm{BS}_{2}$ and $\mathrm{DM} ; k=2 \pi / \lambda$ is the wave number; and $R_{1}, R_{2}$ are the reflectivities of $\mathrm{BS}_{2}$ and $\mathrm{DM}$, respectively. Assuming that the surface displacement error is small, i.e., $|\Delta d| \ll \lambda$, Eq. (1) yields: $\Delta I \cong \gamma \Delta d$, where $\gamma=I_{0} \partial R\left(d_{0}\right) / \partial d$ is the interferometer sensitivity at $d_{0}$. The inset in Fig. 5 illustrates intensity variation with $\Delta d$. The sensitivity parameter $\gamma$ can be calibrated by applying a low-frequency AC voltage with the amplitude $\Delta U$ to the selected PZT actuator at $F \ll F_{t h}$. Taking into account that $d_{0}$ linearly
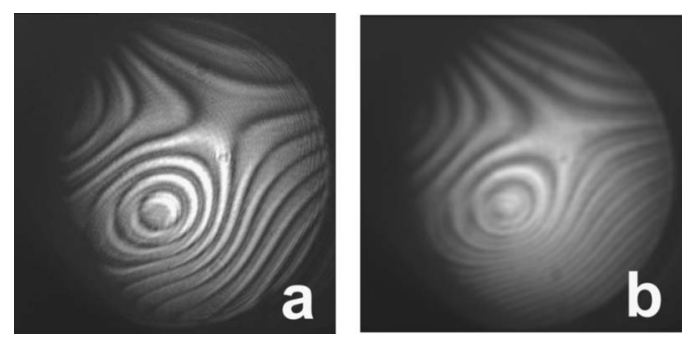

Fig. 3 Averaged over 1-s period interferograms of the piezo DM at (a) low-refresh frequency $F=5 \mathrm{~Hz}$ and (b) high-refresh frequency $F=1700 \mathrm{~Hz}$, above the threshold value $F_{t h} \sim 1500 \mathrm{~Hz}$. 


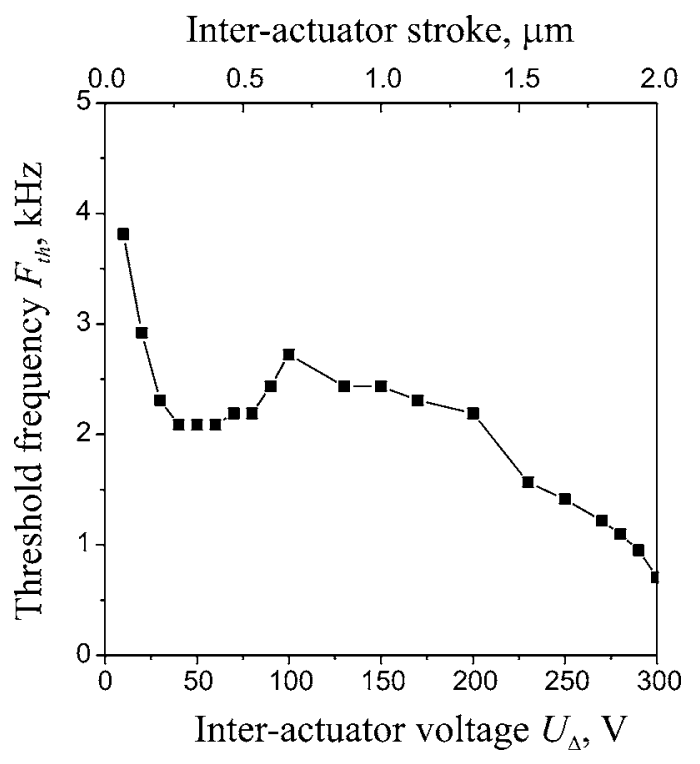

Fig. 4 Threshold multiplexing frequency for the 12-channel DM versus interactuator voltage (stroke).

changes with applied voltage, we obtain: $\gamma=2 U_{\lambda / 2} \Delta I /(\lambda \Delta U)$, where $U_{\lambda / 2}$ is the voltage required for a $\lambda / 2$ shift (see inset in Fig. 5). In our case, $U_{\lambda / 2}$ is measured to be $\sim 38 \mathrm{~V}$. Using these relations, the required surface displacement error $\Delta d$ can be evaluated.

The experimental dependence of $\Delta d$ on $F$ is shown in Fig. 5. In this case, a voltage of $20 \mathrm{~V}$ is applied to the DM channel 8 while multiplexing. As seen, the displacement error does not exceed $\sim \lambda / 100 \mathrm{rms}$ at multiplexing frequencies of less than $3.5 \mathrm{kHz}$. Higher multiplexing frequencies result in the loss of the DM figure stability.

An important advantage of multiplexing control is its scalability to $10^{2}-10^{4} \mathrm{DM}$ channels. This is of particular interest for piezoelectric DMs that employ low-capacitance actuators $(\sim 10 \mathrm{nF})$ operating at frequencies $<1 \mathrm{kHz}$. In this case, low-power switching electronics generating low heat can be integrated with the mirrors. Actually, for our system the average power $\left(W_{d}\right)$ dissipated by a single switch can be estimated as: $F\left[t_{o n} I_{o n}^{2}(\partial U / \partial I)\right.$ $\left.+t_{o f f} I_{o f f}^{2}(\partial U / \partial I)\right]$, where $I_{o n}, I_{o f f}$ are the charging and discharging currents, respectively; $t_{o n}, t_{o f f}$ are the switching times (see Fig. 2); and $\partial U / \partial I$ is the optotriac output resistance. For an actuator operated with a maximum voltage of $300 \mathrm{~V}$ at $F=700 \mathrm{~Hz}, t_{\text {on }} \cong 8 \mu \mathrm{s}, t_{\text {off }} \cong 30 \mu \mathrm{s}$, we obtain $W_{d} \cong 9 \mathrm{~mW}$. With these parameters, the power $W_{P Z T}$ generated in the actuator is $\pi F C_{a} V_{r m s}^{2} \tan \delta \cong 12 \mathrm{~mW}$, for a loss factor $\tan \delta \sim 0.01$. Thus, the heat produced by the switch is lower than the actuator dissipation. The example above gives the maximum estimate for the heat dissipation; practical numbers will be at least two orders of magnitude lower. As discussed by Aldrich, ${ }^{2}$ the multiplexing of highcapacitance actuators has no significant advantages over direct control.

In conclusion, we present the adaptive multiplexing control for piezoelectric deformable mirrors. The adaptive addressing of actuators through the program-based lookup table is implemented to obtain a stable DM figure at a high

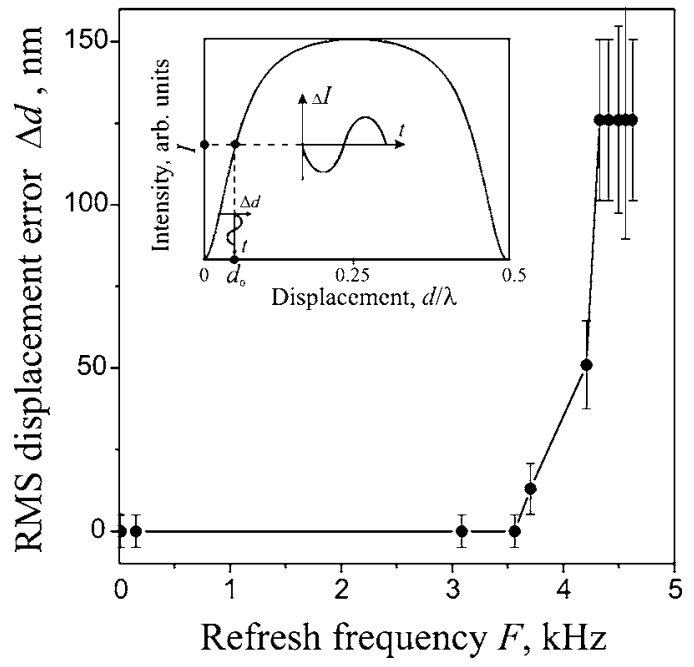

Fig. 5 Surface displacement error of the DM versus multiplexing frequency. Inset shows the reflected intensity from the interferometer as a function of its length $d$.

bandwidth. This approach allows considerable reduction in the cost and size of the driving electronics and the complexity of DM interconnections. The multiplexing has been demonstrated using a 12-channel DM. A high figure stability of $\sim \lambda / 100 \mathrm{rms}$ is obtained for the multiplexing frequencies up to $3.5 \mathrm{kHz}$. Although system performance is slightly less than that reported by Kibblewhite et al. ${ }^{10}$ (they presented a 59-actuator DM driven by four HVAs with a $1-\mu \mathrm{m}$ stroke at a multiplexing frequency of $4 \mathrm{kHz}$ ), the multiplexing electronics are essentially compact, less complicated, and inexpensive (total cost is about \$50). It should be noted that, although the DM multiplexers are now commercially available from Xinetics, Inc., they are still expensive and cannot be integrated with DMs.

\section{References}

1. R. K. Tyson, Principles of Adaptive Optics, Chap. 6, Academic Press (1998).

2. R. K. Tyson Ed., Adaptive Optics Engineering Handbook, Chaps. 2,5,7, and 8, Marcel Dekker New York (2000).

3. R. Bartels, S. Backus, E. Zeek, L. Misoguti, G. Vdovin, I. P. Christov, M. M. Murnane, and H. C. Kapteyn, "Shaped-pulse optimization of coherent emission of high-harmonic soft X-rays," Nature (London) 406, 164-166 (2000).

4. P. A. Knutsson and M. Owner-Petersen, "Emulation of dualconjugate adaptive optics on an 8-m class telescope," Opt. Express 11(18), 2231-2237 (2003).

5. Adaptive optics for industry and medicine, in Proceedings of the 4th International Workshop Series: Springer Proceedings in Physics, Vol. 102, U. Wittrock, Ed. (2005).

6. E. J. Fernandez, I. Iglesias, and P. Artal, "Closed-loop adaptive optics in the human eye," Opt. Lett. 26(10), 746-748 (2001).

7. G. Vdovin and V. Kiyko, "Intracavity control of a 200-W continuouswave NdYAG laser by a micromachined deformable mirror," Opt. Lett. 26(11), 798-800 (2001).

8. G. Vdovin and M. Loktev, "Deformable mirror with thermal actuators," Opt. Lett. 27(9), 677-679 (2002).

9. J. R. Kuhn, G. Moretto, R. Racine, F. Roddier, and R. Coulter, "Concepts for a large-aperture, high dynamic range Telescope," Publ. Astron. Soc. Pac. 113, 1486-1510 (2001).

10. E. Kibblewhite, M. F. Smutko, and M. Chun, "Deformable mirrors for astronomy," in Active and Adaptive Optical Components and Systems II, M. A. Ealey, Ed., Proc. SPIE 1920, 115-120 (1993).

11. http://www.okotech.com.

12. S. J. B. Yoo, M. A. Koza, R. Bhat, and C. Caneau, " $1.5 \mu \mathrm{m}$ symmetric Fabry-Perot modulators with two distinct modulation and chirp characteristics," Appl. Phys. Lett. 72(25), 3246-3248 (1998). 